\title{
Square-like functions generated by the Laplace-Bessel differential operator
}

\author{
Şeyda Keleş ${ }^{*}$ and Simten Bayrakçı
}

"Correspondence:

seydaaltinkol@gmail.com

Department of Mathematics,

Faculty of Science, Akdeniz

University, Antalya, Turkey

\begin{abstract}
We introduce a wavelet-type transform associated with the Laplace-Bessel differential operator $\Delta_{B}=\sum_{k=1}^{n} \frac{\partial^{2}}{\partial x_{k}}+\frac{2 v_{k}}{\partial x_{k}} \frac{\partial}{\partial x_{k}}$ and the relevant square-like functions. An analogue of the Calderón reproducing formula and the $L_{2, v}$ boundedness of the square-like functions are obtained.

MSC: 47G10; 42C40; 44A35
\end{abstract}

Keywords: square functions; generalized translation; wavelet transform; Calderón reproducing formula

\section{Introduction}

The classical square functions $f(x) \rightarrow S_{\varphi}(x)=\left(\int_{0}^{\infty}\left|\left(f * \varphi_{t}\right)(x)\right|^{2} \frac{d t}{t}\right)^{\frac{1}{2}}$, where $\varphi \in S, S \equiv S\left(\mathbb{R}^{n}\right)$ is the Schwartz test function space and $\int_{\mathbb{R}^{n}} \varphi(x) d x=0, \varphi_{t}(x)=t^{-n} \varphi\left(t^{-1} x\right), t>0$, play important role in harmonic analysis and its applications; see Stein [1]. There are a lot of diverse variants of square functions and their applications; see Daly and Phillips [2], Jones et al. [3], Pipher [4], Kim [5]. Square-like functions generated by a composite wavelet transform and its $L_{2}$ estimates are proved by Aliev and Bayrakci [6].

Note that the Laplace-Bessel differential operator $\Delta_{B}$ is known as an important operator in analysis and its applications. The relevant harmonic analysis, known as FourierBessel harmonic analysis associated with the Bessel differential operator $B_{t}=\frac{d^{2}}{d t^{2}}+\frac{2 v}{t} \frac{d}{d t}$, has been the research area for many mathematicians such as Levitan, Muckenhoupt, Stein, Kipriyanov, Klyuchantsev, Löfström, Peetre, Gadjiev, Aliev, Guliev, Triméche, Rubin and others (see [7-14]). Moreover, a lot of mathematicians studied a Calderón reproducing formula. For example, Amri and Rachdi [15], Guliyev and Ibrahimov [16], Kamoun and Mohamed [17], Pathak and Pandey [18], Mourou and Trimèche [19, 20] and others.

In this paper, firstly we introduce a wavelet-like transform associated with the LaplaceBessel differential operator,

$$
\Delta_{B}=\sum_{k=1}^{n} \frac{\partial^{2}}{\partial x_{k}^{2}}+\frac{2 v_{k}}{\partial x_{k}} \frac{\partial}{\partial x_{k}}, \quad v=\left(v_{1}, v_{2}, \ldots, v_{n}\right), v>0
$$

and then the relevant square-like function. The plan of the paper is as follows. Some necessary definitions and auxiliary facts are given in Section 2. In Section 3 we prove a Calderóntype reproducing formula and the $L_{2, v}$ boundedness of the square-like functions.

○2014 Keleş and Bayrakçı; licensee Springer. This is an Open Access article distributed under the terms of the Creative Commons Attribution License (http://creativecommons.org/licenses/by/2.0), which permits unrestricted use, distribution, and reproduction in any medium, provided the original work is properly cited. 


\section{Preliminaries}

$\mathbb{R}_{+}^{n}=\left\{x=\left(x_{1}, \ldots, x_{n}\right) \in \mathbb{R}^{n}: x_{1}>0, x_{2}>0, \ldots, x_{n}>0\right\}$ and let $S\left(\mathbb{R}_{+}^{n}\right)$ be the Schwartz space of infinitely differentiable and rapidly decreasing functions.

$L_{p, v}=L_{p, v}\left(\mathbb{R}_{+}^{n}\right)\left(1 \leq p<\infty, v=\left(v_{1}, \ldots, v_{n}\right) ; v_{1}>0, \ldots, v_{n}>0\right)$ space is defined as the class of measurable functions $f$ on $\mathbb{R}_{+}^{n}$ for which

$$
\|f\|_{p, v}=\left(\int_{\mathbb{R}_{+}^{n}}|f(x)|^{p} x^{2 v} d x\right)^{\frac{1}{p}}<\infty, \quad x^{2 v} d x=x_{1}^{2 v_{1}} x_{2}^{2 v_{2}} \cdots x_{n}^{2 v_{n}} d x_{1} d x_{2} \cdots d x_{n}
$$

In the case $p=\infty$, we identify $L_{\infty} \equiv L_{\infty, v}$ with $C_{0}$ the space of continuous functions vanishing at infinity, and set $\|f\|_{\infty}=\sup _{x \in \mathbb{R}_{+}^{n}}|f(x)|$.

The Fourier-Bessel transform and its inverse are defined by

$$
\begin{aligned}
& f^{\wedge}(x)=F_{\nu}(f)(x)=\int_{\mathbb{R}_{+}^{n}} f(y)\left(\prod_{k=1}^{n} j_{v_{k}-\frac{1}{2}}\left(x_{k} y_{k}\right)\right) y^{2 v} d y, \\
& F_{v}^{-1}(f)(x)=c_{v}(n)\left(F_{v} f\right)(x), \quad c_{v}(n)=\left[2^{2 n} \prod_{k=1}^{n} \Gamma^{2}\left(v_{k}+\frac{1}{2}\right)\right]^{-1},
\end{aligned}
$$

where $j_{v-\frac{1}{2}}$ is the normalized Bessel function, which is also the eigenfunction of the Bessel operator $B_{t}=\frac{d^{2}}{d t^{2}}+\frac{2 v}{t} \frac{d}{d t} ; j_{v-\frac{1}{2}}(0)=1$ and $j_{v-\frac{1}{2}}^{\prime}(0)=0$ (see [10]).

Denote by $T^{y}\left(y \in \mathbb{R}_{+}^{n}\right)$ the generalized translation operator acting according to the law:

$$
\begin{aligned}
T^{y} f(x)= & \pi^{-n / 2} \prod_{k=1}^{n} \Gamma\left(v_{k}+\frac{1}{2}\right) \Gamma^{-1}\left(v_{k}\right) \int_{0}^{\pi} \cdots \int_{0}^{\pi} f\left(\sqrt{x_{1}^{2}-2 x_{1} y_{1} \cos \alpha_{1}+y_{1}^{2}}, \ldots,\right. \\
& \left.\sqrt{x_{n}^{2}-2 x_{n} y_{n} \cos \alpha_{n}+y_{n}^{2}}\right) \prod_{k=1}^{n} \sin ^{2 v_{k}-1} \alpha_{k} d \alpha_{1} \cdots d \alpha_{n} .
\end{aligned}
$$

$T^{y}$ is closely connected with the Bessel operator $B_{t}$ (see [10]). It is known that (see [11])

$$
\begin{aligned}
& \left\|T^{y} f\right\|_{p, v} \leq\|f\|_{p, v} \quad \forall y \in \mathbb{R}_{+}^{n}, 1 \leq p \leq \infty \\
& \left\|T^{y} f-f\right\|_{p, v} \rightarrow 0, \quad|y| \rightarrow 0,1 \leq p \leq \infty .
\end{aligned}
$$

The generalized convolution ' $B$-convolution' associated with the generalized translation operator is $(f * g)(x)=\int_{\mathbb{R}_{n}^{+}} f(y)\left(T^{y} g(x)\right) y^{2 v} d y$ for which

$$
(f * g)^{\wedge}=f^{\wedge} g^{\wedge} .
$$

We consider the $B$-maximal operator (see $[8,21]$ )

$$
M_{B} f(x)=\sup _{r>0}\left|E_{+}(0, r)\right|_{2 v}^{-1} \int_{E_{+}(0, r)} T^{y}|f(x)| y^{2 v} d y
$$

where $E_{+}(0, r)=\left\{y \in \mathbb{R}_{+}^{n}:|y|<r\right\}$ and $\left|E_{+}(0, r)\right|_{2 v}=\int_{E_{+}(0, r)} x^{2 v} d x=C r^{n+2 v}$. Moreover, the following inequalities are satisfied (see for details [22]). 
(a) If $f \in L_{1, v}\left(\mathbb{R}_{+}^{n}\right)$, then for every $\alpha>0$,

$$
\left|\left\{x: M_{B} f(x)>\alpha\right\}\right|_{2 v} \leq \frac{c}{\alpha} \int_{\mathbb{R}_{+}^{n}}|f(x)| x^{2 v} d x,
$$

where $c>0$ is independent of $f$.

(b) If $f \in L_{p, v}\left(\mathbb{R}_{+}^{n}\right), 1<p \leq \infty$, then $M_{B} f \in L_{p, v}\left(R_{+}^{n}\right)$ and

$$
\left\|M_{B} f\right\|_{p, v} \leq C_{p}\|f\|_{p, v}
$$

where $c_{p}$ is independent of $f$.

Furthermore, if $f \in L_{p, v}\left(\mathbb{R}_{+}^{n}\right), 1 \leq p \leq \infty$, then

$$
\lim _{r \rightarrow 0}\left|E_{+}(0, r)\right|_{2 v}^{-1} \int_{E_{+}(0, r)} T^{y} f(x) y^{2 v} d y=f(x) .
$$

Now, we will need the generalized Gauss-Weierstrass kernel defined as

$$
g_{v}(x, t)=F_{v}^{-1}\left(e^{-t|\cdot|^{2}}\right)(x)=\sqrt{c_{v}(n)} t^{\frac{-(n+2|v|)}{2}} e^{\frac{-x^{2}}{4 t}}, \quad x \in \mathbb{R}_{+}^{n}, t>0
$$

$c_{v}(n)$ being defined by $(2.2)$ and $|v|=v_{1}+v_{2}+\cdots+v_{n}$.

The kernel $g_{v}(x, t)$ possesses the following properties:
(a) $\quad F_{v}\left(g_{v}(\cdot, t)\right)(x)=e^{-t|x|^{2}} \quad(t>0)$;
(b) $\int_{\mathbb{R}_{+}^{n}} g_{\nu}(y, t) d y=1 \quad(t>0)$.

Given a function $f: \mathbb{R}_{n}^{+} \rightarrow \mathbb{C}$, the generalized Gauss-Weierstrass semigroup, $G_{t} f(x)$ is defined as

$$
G_{t} f(x)=\int_{\mathbb{R}_{+}^{n}} g_{v}(y, t)\left(T^{y} f(x)\right) y^{2 v} d y, \quad t>0 .
$$

This semigroup is well known and arises in the context of stable random processes in probability, in pseudo-differential parabolic equations and in integral geometry; see Koldobsky, Landkof, Fedorjuk, Aliev, Rubin, Sezer and Uyhan (see [23-26]).

The following lemma contains some properties of the semigroup $\left\{G_{t} f\right\}_{t \geq 0}$. (Compare with the analogous properties of the classical Gauss-Weierstrass integral $[1,27,28]$.)

Lemma 2.1 If $\in L_{p, v}, 1 \leq p \leq \infty\left(L_{\infty} \equiv C_{0}\right)$, then
(a) $\left\|G_{t} f\right\|_{p, v} \leq c\|f\|_{p, v}$,
(b) $\lim _{t \rightarrow 0} G_{t} f(x)=f(x)$.

The limit is understood in $L_{p, v}$ norm and pointwise almost all $x \in \mathbb{R}_{+}^{n}$. If $f \in C_{0}$, then the limit is uniform on $\mathbb{R}_{+}^{n}$.

(c) $\sup _{t>0}\left|G_{t} f(x)\right| \leq c M_{B} f(x)$

where $M_{B} f$ is the well-known Hardy-Littlewood maximal function. 
Moreover, let $h(z)$ be an absolutely continuous function on $[0, \infty)$ and

$$
\alpha=\int_{0}^{\infty} \frac{h(z)}{z} d z<\infty .
$$

If we denote $w(z)=h^{\prime}(z)$, we have from $(2.13)$

$$
h(0)=0 \text { and } \quad h(\infty)=0
$$

(see for details [29]).

Now, we define the following wavelet-like transform:

$$
V_{t} f(x)=\frac{1}{\alpha} \int_{0}^{\infty} G_{t z} f(x) w(z) d z
$$

where $w(z)$ is known as 'wavelet function', $\int_{0}^{\infty} w(z) d z=0$, and the function $G_{t z} f(x)$ is the generalized Gauss-Weierstrass semigroup.

Using wavelet-like transform (2.15), we define the following square-like functions:

$$
(S f)(x)=\left(\int_{0}^{\infty}\left|V_{t} f(x)\right|^{2} \frac{d t}{t}\right)^{\frac{1}{2}} .
$$

\section{Main theorems and proofs}

\section{Theorem 3.1}

(a) Letf $\in L_{p, v}, 1 \leq p \leq \infty\left(L_{\infty} \equiv C_{0}\right)$, $v>0$. We have

$$
\left\|V_{t} f\right\|_{p, v} \leq c_{1} c_{2}\|f\|_{p, v} \quad(\forall t>0),
$$

where $c_{1}=2^{2|v|-n},|v|=v_{1}+v_{2}+\cdots+v_{n}, c_{2}=\frac{1}{\alpha} \int_{0}^{\infty}|w(z)| d z<\infty$.

(b) Let $f \in L_{p, v}, 1<p \leq \infty\left(L_{\infty} \equiv C_{0}\right)$. We have

$$
\int_{0}^{\infty} V_{t} f(x) \frac{d t}{t} \equiv \lim _{\substack{\epsilon \rightarrow 0 \\ \rho \rightarrow \infty}} \int_{\epsilon}^{\rho} V_{t} f(x) \frac{d t}{t}=f(x),
$$

where limit can be interpreted in the $L_{p, v}$ norm and pointwise for almost all $x \in \mathbb{R}_{+}^{n}$. If $f \in C_{0}$, the convergence is uniform on $\mathbb{R}_{+}^{n}$.

Theorem 3.2 If $\in L_{2, v}$, then

$$
\|S f\|_{2, v} \leq \frac{1}{2}\|f\|_{2, v}
$$

Proof of Theorem 3.1 (a) By using the Minkowski inequality, we have

$$
\begin{aligned}
\left\|V_{t} f\right\|_{p, v} & =\frac{1}{\alpha}\left(\int_{\mathbb{R}_{+}^{n}}\left|\int_{0}^{\infty} G_{t z} f(x) w(z) d z\right|^{p} x^{2 v} d x\right)^{\frac{1}{p}} \\
& \leq \frac{1}{\alpha} \int_{0}^{\infty}|w(z)|\left\|G_{t z} f\right\|_{p, v} d z
\end{aligned}
$$




$$
\begin{aligned}
\left\|G_{t z} f\right\|_{p, v} & =\left(\int_{\mathbb{R}_{+}^{n}}\left|\int_{\mathbb{R}_{+}^{n}} g_{v}(y, t z) T^{y} f(x) y^{2 v} d y\right|^{p} x^{2 v} d x\right)^{\frac{1}{p}} \\
& \leq \int_{\mathbb{R}_{+}^{n}}\left|g_{v}(y, t z)\right|\left(\int_{\mathbb{R}_{+}^{n}}\left|T^{y} f(x)\right|^{p} x^{2 v} d x\right)^{\frac{1}{p}} y^{2 v} d y \\
& \leq\|f\|_{p, v} \int_{\mathbb{R}_{+}^{n}}\left|g_{v}(y, t z)\right| y^{2 v} d y=c_{1}\|f\|_{p, v} .
\end{aligned}
$$

Taking into account the following equality for $\operatorname{Re} \mu>0, \operatorname{Re} v>0, p>0$ (see [30, p.370])

$$
\int_{0}^{\infty} x^{\nu-1} e^{-\mu x^{p}} d x=\frac{1}{p} \mu^{-\frac{\nu}{p}} \Gamma\left(\frac{v}{p}\right)
$$

we have

$$
\int_{0}^{\infty} x^{2 v} e^{-x^{2}} d x=\frac{1}{2} \Gamma\left(v+\frac{1}{2}\right), \quad v>0
$$

in one dimension. By using this equality, we get

$$
\begin{aligned}
c_{1} & =\int_{\mathbb{R}_{+}^{n}}\left|g_{\nu}(y, t)\right| y^{2 v} d y \\
& =2^{-n} \prod_{k=1}^{n} \Gamma^{-1}\left(v_{k}+\frac{1}{2}\right) t^{\frac{-(n+2|v|)}{2}} \int_{\mathbb{R}_{+}^{n}} e^{-\frac{|y|^{2}}{4 t}} y^{2 v} d y \quad\left(y=2 \sqrt{t} y, d y=2^{n} t^{\frac{n}{2}} d y\right) \\
& =2^{-n} \prod_{k=1}^{n} \Gamma^{-1}\left(v_{k}+\frac{1}{2}\right) t^{\frac{-(n+2|v|)}{2}} \int_{\mathbb{R}_{+}^{n}} e^{-|y|^{2}} 2^{2|v|} t^{|v|} 2^{n} t^{\frac{n}{2}} y^{2 v} d y \\
& =2^{2|v|} \prod_{k=1}^{n} \Gamma^{-1}\left(v_{k}+\frac{1}{2}\right) \int_{\mathbb{R}_{+}^{n}} e^{-|y|^{2}} y^{2 v} d y \\
& =2^{2|v|} \prod_{k=1}^{n} \Gamma^{-1}\left(v_{k}+\frac{1}{2}\right) \prod_{k=1}^{n} \Gamma\left(v_{k}+\frac{1}{2}\right) 2^{-n} \\
& =2^{2|v|-n} .
\end{aligned}
$$

So we have $\left\|G_{t z} f\right\|_{p, v} \leq 2^{2|v|-n}\|f\|_{p, v}$, and then inequality (3.1).

(b) Let $\left(A_{\epsilon, \rho} f\right)(x)=\int_{\epsilon}^{\rho} V_{t} f(x) \frac{d t}{t}, 0<\epsilon<\rho<\infty$. Applying Fubini's theorem, we get

$$
\begin{aligned}
\left(A_{\epsilon, \rho} f\right)(x) & =\frac{1}{\alpha} \int_{\epsilon}^{\rho}\left(\int_{0}^{\infty} G_{t z} f(x) w(z) d z\right) \frac{d t}{t} \\
& =\frac{1}{\alpha} \int_{0}^{\infty} w(z)\left(\int_{\epsilon}^{\rho} G_{t z} f(x) \frac{d t}{t}\right) d z \\
& =\frac{1}{\alpha} \int_{0}^{\infty} w(z)\left(\int_{\epsilon z}^{\rho z} G_{t} f(x) \frac{d t}{t}\right) d z \\
& =\frac{1}{\alpha} \int_{0}^{\infty}\left(\int_{\frac{t}{\rho}}^{\frac{t}{\epsilon}} w(z) d z\right) G_{t} f(x) \frac{d t}{t}
\end{aligned}
$$




$$
\begin{aligned}
& =\frac{1}{\alpha} \int_{0}^{\infty} \frac{1}{t}\left[h\left(\frac{t}{\epsilon}\right)-h\left(\frac{t}{\rho}\right)\right] G_{t} f(x) d t \\
& =\frac{1}{\alpha} \int_{0}^{\infty} \frac{h(t)}{t} G_{\epsilon t} f(x) d t-\frac{1}{\alpha} \int_{0}^{\infty} \frac{h(t)}{t} G_{\rho t} f(x) d t \\
& =\left(A_{\epsilon} f\right)(x)-\left(A_{\rho} f\right)(x) .
\end{aligned}
$$

By Theorem 1.15 in $[28, \mathrm{p} .3]$, if $1<p \leq \infty\left(L_{\infty} \equiv C_{0}\right)$, then

$$
\lim _{\rho \rightarrow \infty}\left\|G_{\rho t} f\right\|_{p, v}=0
$$

Therefore, by the Minkowski inequality and the Lebesgue dominated convergence theorem, taking into account Lemma 2.1, we have

$$
\begin{aligned}
\left\|A_{\rho} f\right\|_{p, v} & =\frac{1}{\alpha}\left(\int_{\mathbb{R}_{n}^{+}}\left(\int_{0}^{\infty} \frac{h(t)}{t} G_{\rho t} f(x) d t\right)^{p} x^{2 v} d x\right)^{\frac{1}{p}} \\
& \leq \frac{1}{\alpha} \int_{0}^{\infty} \frac{h(t)}{t}\left\|G_{\rho t} f\right\|_{p, v} d t \\
& =\frac{1}{\alpha} \int_{0}^{\infty} \frac{h\left(\frac{t}{\rho}\right)}{\frac{t}{\rho}}\left\|G_{\rho t} f\right\|_{p, v} \frac{1}{\rho} d t \rightarrow 0, \quad \rho \rightarrow \infty
\end{aligned}
$$

and

$$
\begin{aligned}
\left\|A_{\epsilon} f-f\right\|_{p, v} & =\left(\int_{\mathbb{R}_{n}^{+}}\left(\frac{1}{\alpha} \int_{0}^{\infty} \frac{h(t)}{t} G_{\epsilon} f(x) d t-f(x)\right)^{p} x^{2 v} d x\right)^{\frac{1}{p}} \\
\stackrel{(2.13)}{=} & \left(\int_{\mathbb{R}_{n}^{+}}\left(\frac{1}{\alpha} \int_{0}^{\infty} \frac{h(t)}{t} G_{\epsilon t} f(x) d t-\frac{1}{\alpha} \int_{0}^{\infty} \frac{h(t)}{t} f(x) d t\right)^{p} x^{2 v} d x\right)^{\frac{1}{p}} \\
& \leq \frac{1}{\alpha} \int_{0}^{\infty} \frac{h(t)}{t}\left\|G_{\epsilon} f-f\right\|_{p, v} d t \rightarrow 0, \quad \epsilon \rightarrow 0 .
\end{aligned}
$$

Finally, for $1<p \leq \infty\left(L_{\infty} \equiv C_{0}\right)$, we get

$$
\left\|A_{\epsilon, \rho} f-f\right\|_{p, v}=\left\|A_{\epsilon} f-f\right\|_{p, v}+\left\|A_{\rho} f\right\|_{p, v} \rightarrow 0, \quad \epsilon \rightarrow 0, \rho \rightarrow \infty .
$$

The a.e. convergence is based on the standard maximal function technique (see [31, p.60], [29] and [32]).

Proof of Theorem 3.2 Firstly, let $f \in S\left(\mathbb{R}_{+}^{n}\right)$. By making use of the Fubini and Plancherel (for Fourier-Bessel transform) theorems, we get

$$
\begin{aligned}
\|S f\|_{2, v}^{2} & =\int_{\mathbb{R}_{+}^{n}}\left(\int_{0}^{\infty}\left|V_{t} f(x)\right|^{2} \frac{d t}{t}\right) x^{2 v} d x \\
& =\int_{0}^{\infty}\left(\int_{\mathbb{R}_{n}^{+}}\left|V_{t} f(x)\right|^{2} x^{2 v} d x\right) \frac{d t}{t} \\
& =\int_{0}^{\infty}\left(\int_{\mathbb{R}_{n}^{+}}\left|\left(V_{t} f\right)^{\wedge}(x)\right|^{2} x^{2 v} d x\right) \frac{d t}{t}
\end{aligned}
$$


Keleş and Bayrakçı Advances in Difference Equations 2014, 2014:281

Page 7 of 9

http://www.advancesindifferenceequations.com/content/2014/1/281

and

$$
\begin{aligned}
\left(V_{t} f\right)^{\wedge}(x) & =F_{v}\left(V_{t} f\right)(x)=\frac{1}{\alpha} \int_{\mathbb{R}_{n}^{+}}\left(\int_{0}^{\infty} G_{t z} f(y) w(z) d z\right) \prod_{k=1}^{n} j_{v_{k}-\frac{1}{2}}\left(x_{k} y_{k}\right) y^{2 v} d y \\
& =\frac{1}{\alpha} \int_{0}^{\infty} w(z)\left(\int_{\mathbb{R}_{n}^{+}} G_{t z} f(y) \prod_{k=1}^{n} j_{v_{k}-\frac{1}{2}}\left(x_{k} y_{k}\right) y^{2 v} d y\right) d z \\
& =\frac{1}{\alpha} \int_{0}^{\infty} w(z)\left(G_{t z} f\right)^{\wedge}(x) d z \\
& \stackrel{(2.5)}{=} \frac{1}{\alpha} \int_{0}^{\infty} w(z) f^{\wedge}(x) e^{-t z|x|^{2}} d z .
\end{aligned}
$$

Now, by using Fubini's theorem, we have

$$
\begin{aligned}
\|S f\|_{2, v}^{2}= & \frac{1}{\alpha^{2}} \int_{0}^{\infty}\left[\int_{\mathbb{R}_{n}^{+}}\left(f^{\wedge}(x)\right)^{2}\left(\int_{0}^{\infty} w(z) e^{-t z|x|^{2}} d z\right)^{2} x^{2 v} d x\right] \frac{d t}{t} \\
= & \frac{1}{\alpha^{2}} \int_{\mathbb{R}_{n}^{+}}\left(f^{\wedge}(x)\right)^{2} \int_{0}^{\infty} \frac{d t}{t}\left(\int_{0}^{\infty} w(z) e^{-t z|x|^{2}} d z\right)^{2} x^{2 v} d x \\
& \left(t=\tau|x|^{-2}, d t=|x|^{-2} d \tau\right) \\
= & \frac{1}{\alpha^{2}} \int_{\mathbb{R}_{n}^{+}}\left(f^{\wedge}(x)\right)^{2} \int_{0}^{\infty} \frac{d \tau}{\tau}\left(\int_{0}^{\infty} w(z) e^{-\tau z} d z\right)^{2} x^{2 v} d x \\
= & C^{2} \frac{1}{\alpha^{2}}\|f\|_{2, v}^{2},
\end{aligned}
$$

where

$$
C=\left(\int_{0}^{\infty} \frac{d \tau}{\tau}\left(\int_{0}^{\infty} e^{-\tau z} w(z) d z\right)^{2}\right)^{1 / 2}
$$

Since $w(z)=h^{\prime}(z), h(z) \geq 0, h(\infty)=h(0)=0$, it follows that

$$
\begin{aligned}
C & =\left(\int_{0}^{\infty} \frac{d \tau}{\tau}\left(\int_{0}^{\infty} e^{-\tau z} w(z) d z\right)^{2}\right)^{1 / 2} \\
& =\left(\int_{0}^{\infty}\left(\int_{0}^{\infty} \sqrt{\tau} e^{-\tau z} h(z) d z\right)^{2} d \tau\right)^{1 / 2} \\
& \leq \int_{0}^{\infty} h(z)\left(\int_{0}^{\infty} \tau e^{-2 \tau z} d \tau\right)^{1 / 2} d z \quad(2 z \tau=t, 2 z d \tau=d t) \\
& =\int_{0}^{\infty} h(z)\left(\int_{0}^{\infty} \frac{t}{2 z} e^{-t} \frac{1}{2 z} d t\right)^{1 / 2} d z \\
& =\int_{0}^{\infty} \frac{h(z)}{2 z}\left(\int_{0}^{\infty} t e^{-t} d t\right)^{1 / 2} d z=\frac{1}{2} \alpha .
\end{aligned}
$$


Finally, we get

$$
\|S f\|_{2, v} \leq \frac{1}{2}\|f\|_{2, v}
$$

For arbitrary $f \in L_{2, v}\left(\mathbb{R}_{+}^{n}\right)$, the result follows by density of the class $S\left(\mathbb{R}_{+}^{n}\right)$ in $L_{2, v}\left(\mathbb{R}_{+}^{n}\right)$. Namely, let $\left(f_{n}\right)$ be a sequence of functions in $S\left(\mathbb{R}_{+}^{n}\right)$, which converge to $f$ in $L_{2, v}\left(\mathbb{R}_{+}^{n}\right)$-norm. That is, $\lim _{n \rightarrow \infty}\left\|f_{n}(x)-f(x)\right\|_{2, v}=0, \forall x \in \mathbb{R}_{+}^{n}$.

From the 'triangle inequality' $\left(\left(\|u\|_{2, v}-\|v\|_{2, v}\right)^{2} \leq\|u-v\|_{2, v}^{2}\right)$, we have

$$
\begin{aligned}
\left|\left(S f_{n}\right)(x)-\left(S f_{m}\right)(x)\right|^{2} & =\left[\left(\int_{0}^{\infty}\left|V_{t} f_{n}(x)\right|^{2} \frac{d t}{t}\right)^{\frac{1}{2}}-\left(\left(\int_{0}^{\infty}\left|V_{t} f_{m}(x)\right|^{2} \frac{d t}{t}\right)^{\frac{1}{2}}\right)\right]^{2} \\
& \leq \int_{0}^{\infty}\left|V_{t} f_{n}(x)-V_{t} f_{m}(x)\right|^{2} \frac{d t}{t} \\
& =\int_{0}^{\infty}\left|V_{t}\left(f_{n}-f_{m}\right)\right|^{2} \frac{d t}{t} \\
& =\left(S\left(f_{n}-f_{m}\right)(x)\right)^{2} .
\end{aligned}
$$

Hence

$$
\left\|S f_{n}-S f_{m}\right\|_{2, v} \leq\left\|S\left(f_{n}-f_{m}\right)\right\|_{2, v} \leq \frac{1}{2}\left\|f_{n}-f_{m}\right\|_{2, \nu}
$$

This shows that the sequence $\left(S f_{n}\right)$ converges to $S f$ in $L_{2, v}\left(\mathbb{R}_{+}^{n}\right)$-norm. Thus

$$
\|S f\|_{2, v} \leq \frac{1}{2}\|f\|_{2, v}, \quad \forall f \in L_{2, v}\left(\mathbb{R}_{+}^{n}\right)
$$

and the proof is complete.

\section{Competing interests}

The authors declare that they have no competing interests.

\section{Authors' contributions}

All authors contributed equally to this work. All authors read and approved the final manuscript.

\section{Acknowledgements}

The authors would like to thank the referees for their valuable comments. This work was supported by the Scientific Research Project Administration Unit of the Akdeniz University (Turkey).

Received: 24 June 2014 Accepted: 19 October 2014 Published: 31 Oct 2014

\section{References}

1. Stein, EM: Harmonic Analysis: Real-Variable Methods, Orthogonality, and Oscillatory Integrals. Princeton University Press, Princeton (1993)

2. Daly, JE, Phillips, KL: Walsh multipliers and square functions for the Hardy space $H^{1}$. Acta Math. Hung. 79(4), 311-327 (1998)

3. Jones, RL, Ostrovskii, IV, Rosenblatt, JM: Square functions in ergodic theory. Ergod. Theory Dyn. Syst. 16, 267-305 (1996)

4. Pipher, J: Bounded double square functions. Ann. Inst. Fourier (Grenoble) 36(2), 69-82 (1986)

5. Kim, YC: Weak type estimates of square functions associated with quasiradial Bochner-Riesz means on certain Hardy spaces. J. Math. Anal. Appl. 339(1), 266-280 (2008)

6. Aliev, IA, Bayrakci, S: Square-like functions generated by a composite wavelet transform. Mediterr. J. Math. 8, 553-561 (2011)

7. Gadjiev, AD, Aliev, IA: On a class of potential type operator generated by a generalized shift operator. In: Reports of Enlarged Session of the Seminars of I. N. Vekua Inst. Appl. Math. (Tbilisi), vol. 3(2), pp. 21-24 (1988) (in Russian) 
8. Guliev, VS: Sobolev's theorem for Riesz B-potentials. Dokl. Akad. Nauk SSSR 358(4), 450-451 (1998) (in Russian)

9. Kipriyanov, IA, Klyuchantsev, MI: On singular integrals generated by the generalized shift operator II. Sib. Mat. Zh. 11, 1060-1083 (1970)

10. Levitan, BM: Expansion in Fourier series and integrals in Bessel functions. Usp. Mat. Nauk 6, 102-143 (1951) (in Russian)

11. Löfström, J, Peetre, J: Approximation theorems connected with generalized translations. Math. Ann. 181, 255-268 (1969)

12. Muckenhoupt, B, Stein, E: Classical expansions and their relation to conjugate harmonic functions. Trans. Am. Math. Soc. 118, 17-92 (1965)

13. Rubin, B: Intersection bodies and generalized cosine transforms. Adv. Math. 218, 696-727 (2008)

14. Trimèche, K: Generalized Wavelets and Hypergroups. Gordon \& Breach, New York (1997)

15. Amri, B, Rachdi, LT: Calderón reproducing formula for singular partial differential operators. Integral Transforms Spec. Funct. 25(8), 597-611 (2014)

16. Guliyev, VS, Ibrahimov, EJ: Calderón reproducing formula associated with Gegenbauer operator on the half line. J. Math. Anal. Appl. 335(2), 1079-1094 (2007)

17. Komoun, L, Mohamed, S: Calderón's reproducing formula associated with partial differential operators on the half plane. Glob. J. Pure Appl. Math. 2(3), 197-205 (2006)

18. Pathak, RS, Pandey, G: Calderón's reproducing formula for Hankel convolution. Int. J. Math. Sci. 2006, Article ID 24217 (2006)

19. Mourou, MA, Trimèche, $\mathrm{K}$ : Calderón's reproducing formula related to the Dunkl operator on the real line. Monatshefte Math. 136(1), 47-65 (2002)

20. Mourou, MA, Trimèche, K: Calderón's reproducing formula associated with the Bessel operator. J. Math. Anal. Appl. 219(1), 97-109 (1998)

21. Guliyev, VS: Sobolev's theorem for anisotropic Riesz-Bessel potentials on Morrey-Bessel spaces. Dokl. Akad. Nauk SSSR 367(2), 155-156 (1999)

22. Guliyev, VS: On maximal function and fractional integral, associated with the Bessel differential operator. Math. Inequal. Appl. 2, 317-330 (2003)

23. Aliev, IA, Rubin, B, Sezer, S, Uyhan, SB: Composite wavelet transforms: applications and perspectives. In: Radon Transforms, Geometry and Wavelets. Contemporary Mathematics, vol. 464, pp. 1-25. Am. Math. Soc., Providence (2008)

24. Fedorjuk, MV: Asymptotic behavior of the Green function of a pseudodifferential parabolic equation. Differ. Uravn. 14(7), 1296-1301 (1978)

25. Koldobsky, A: Fourier Analysis in Convex Geometry. Mathematical Surveys and Monographs, vol. 116. Am. Math. Soc. Providence (2005)

26. Landkof, NS: Several remarks on stable random processes and $\alpha$-superharmonic functions. Mat. Zametki 14, $901-912$ (1973) (in Russian)

27. Aliev, IA: Bi-parametric potentials, relevant function spaces and wavelet-like transforms. Integral Equ. Oper. Theory 65 151-167 (2009)

28. Rubin, B: Fractional Integrals and Potentials. Pitman Monographs and Surveys in Pure and Applied Mathematics, vol. 82. Longman, Harlow (1996)

29. Aliev, IA, Bayrakci, S: On inversion of Bessel potentials associated with the Laplace-Bessel differential operator. Acta Math. Hung. 95, 125-145 (2002)

30. Gradshtein, IS, Ryzhik, IM: Tables of Integrals, Sums, Series and Products, 5th edn. Academic Press, New York (1994)

31. Stein, EM, Weiss, G: Introduction to Fourier Analysis on Euclidean Spaces. Princeton University Press, Princeton (1971)

32. Aliev, IA, Rubin, B: Wavelet-like transforms for admissible semi-groups; inversion formulas for potentials and Radon transforms. J. Fourier Anal. Appl. 11, 333-352 (2005)

\section{Submit your manuscript to a SpringerOpen ${ }^{\circ}$ journal and benefit from:}

- Convenient online submission

Rigorous peer review

- Immediate publication on acceptance

- Open access: articles freely available online

- High visibility within the field

- Retaining the copyright to your article 